\title{
Monte Carlo simulations of two-dimensional hard core lattice gases
}

Cite as: J. Chem. Phys. 126, 114508 (2007); https://doi.org/10.1063/1.2539141

Submitted: 12 December 2006 . Accepted: 16 January 2007 . Published Online: 21 March 2007

Heitor C. Marques Fernandes, Jeferson J. Arenzon, and Yan Levin

\section{ARTICLES YOU MAY BE INTERESTED IN}

Exact Finite Method of Lattice Statistics. I. Square and Triangular Lattice Gases of Hard Molecules

The Journal of Chemical Physics 45, 2482 (1966); https://doi.org/10.1063/1.1727966

Phase Transitions in Two-Dimensional Lattice Gases of Hard-Square Molecules

The Journal of Chemical Physics 46, 2922 (1967); https://doi.org/10.1063/1.1841157

Phase Transition of a Hard-Core Lattice Gas. The Square Lattice with Nearest-Neighbor Exclusion

The Journal of Chemical Physics 45, 3983 (1966); https://doi.org/10.1063/1.1727448

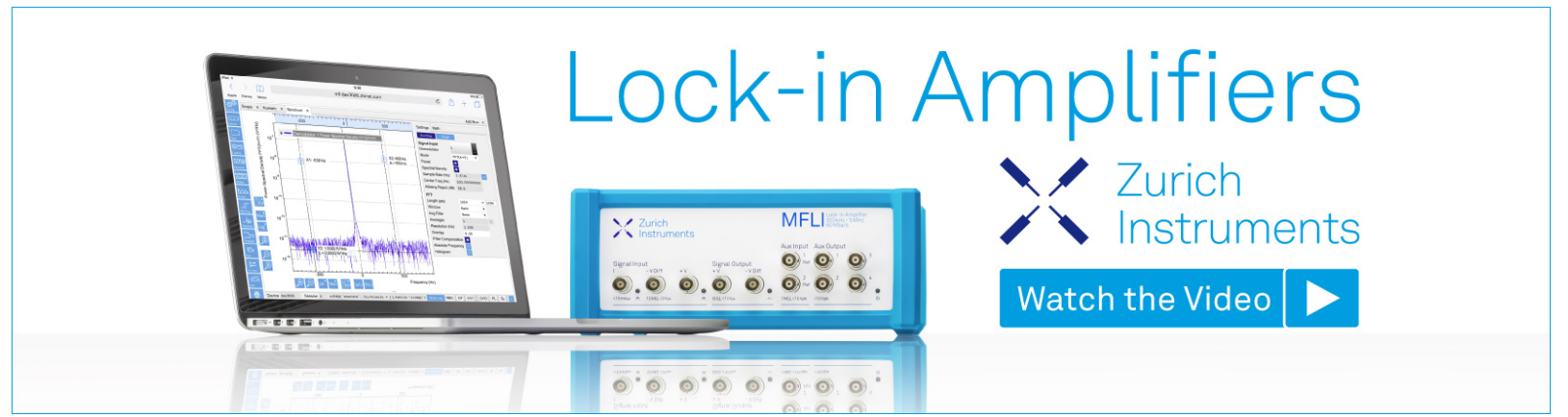

J. Chem. Phys. 126, 114508 (2007); https://doi.org/10.1063/1.2539141 


\title{
Monte Carlo simulations of two-dimensional hard core lattice gases
}

\author{
Heitor C. Marques Fernandes, ${ }^{\text {a) }}$ Jeferson J. Arenzon, ${ }^{\text {b) }}$ and Yan Levin ${ }^{\text {c) }}$ \\ Instituto de Física, Universidade Federal do Rio Grande do Sul, Caixa Postal 15051, 91501-970 Porto \\ Alegre RS, Brazil
}

(Received 12 December 2006; accepted 16 January 2007; published online 21 March 2007)

\begin{abstract}
Monte Carlo simulations are used to study lattice gases of particles with extended hard cores on a two-dimensional square lattice. Exclusions of one and up to five nearest neighbors (NN) are considered. These can be mapped onto hard squares of varying side length, $\lambda$ (in lattice units), tilted by some angle with respect to the original lattice. In agreement with earlier studies, the 1NN exclusion undergoes a continuous order-disorder transition in the Ising universality class. Surprisingly, we find that the lattice gas with exclusions of up to second nearest neighbors (2NN) also undergoes a continuous phase transition in the Ising universality class, while the LandauLifshitz theory predicts that this transition should be in the universality class of the XY model with cubic anisotropy. The lattice gas of $3 \mathrm{NN}$ exclusions is found to undergo a discontinuous order-disorder transition, in agreement with the earlier transfer matrix calculations and the LandauLifshitz theory. On the other hand, the gas of $4 \mathrm{NN}$ exclusions once again exhibits a continuous phase transition in the Ising universality class-contradicting the predictions of the Landau-Lifshitz theory. Finally, the lattice gas of 5NN exclusions is found to undergo a discontinuous phase transition. (C) 2007 American Institute of Physics. [DOI: 10.1063/1.2539141]
\end{abstract}

\section{INTRODUCTION}

Since the early days of statistical mechanics lattice gases serve as the foundation on which many models of complex physical systems are constructed. These range from simple fluids ${ }^{1}$ to structural glasses and granular materials. ${ }^{2}$ The simplest lattice gas consists of noninteracting particles which are constrained to move on a lattice with a restriction that each lattice site is occupied by at most one particle. This model can be solved exactly, showing that the thermodynamics is trivial and no phase transition is present. A lattice gas in which particles interact with their nearest neighbors can be mapped onto the Ising model in external field and exhibits a first order phase transition terminating in a critical point. The difficulty and the scarcity of exact solutions have stimulated the development of various approximate theories aimed at treating more complex interaction potentials. Examples of these are the high and the low temperature (or density) expansions, ${ }^{3}$ generalization of Bethe's methods for Ising model (which became known as cluster variational methods), ${ }^{4}$ as well as the approximation schemes such as Rushbrooke and Scoins. ${ }^{5}$ Since melting is dominated by strong short ranged repulsive forces, a lattice gas in which particles interact exclusively through an extended hard core- a particle on one site prevents the neighboring sites from being occupied-has attracted a particular attention. ${ }^{6}$ In these systems temperature plays no role since the interaction energy is infinite inside the exclusion region and vanishes outside. Surprisingly already with one nearest neighbor exclusion (1NN), an order-disorder transition appears. The

\footnotetext{
${ }^{a}$ Electronic mail: heitor@if.ufrgs.br

${ }^{b)}$ Electronic mail: arenzon@if.ufrgs.br

${ }^{c)}$ Electronic mail: levin@if.ufrgs.br
}

transition is purely entropic and is similar to the freezing of hard spheres, except that now the transition is of second order.

In this paper we are interested in the phase transitions which occur in hardcore lattice gases as the size of the exclusion region is extended. We start with the $1 \mathrm{NN}$ and progressively increase the exclusion region up to five nearest neighbors $(5 \mathrm{NN})$. To study the phase transitions, grandcanonical Monte Carlo simulations are performed with alternating attempts at inclusion/removal of particles followed by some tentative diffusion moves. The grand-canonical ensemble was chosen because it allows for the density fluctuations which are particularly important since the thermal fluctuations are absent in these systems.

The specific question that we would like to address here is how does the order-disorder transition depends on the range of the exclusion region and whether the universality class and the order of the phase transition can be predicted based purely on symmetry considerations.

The paper is organized as follows: Sec. II defines the relevant order parameters characterizing the ordered phases. Section III presents the results of the grand-canonical Monte Carlo simulations and Sec. IV gives the final discussion and the conclusions.

\section{EXCLUSION MODELS, SUBLATTICES, AND ORDER PARAMETERS}

A lattice gas is composed of particles whose positions are restricted to coincide with the vertices of a given lattice, and where each site can be occupied by, at most, one particle. When there is no further interaction between the particles, this system presents a trivial thermodynamics and no phase transition. Instead, here we consider equilibrium lattice gases 
where the range of exclusion extends over the neighboring sites. Increasing the radius of the exclusion shell is equivalent to taking smaller lattice cell sizes as one goes to the continuum limit. These exclusion shells may be interpreted in terms of geometric hard core particles. All cases considered here are equivalent to parallel hard squares of size $\lambda$, either tilted or not. Since hard core potentials allow either zero or infinite energies, temperature is not a relevant parameter, the systems thus being athermal. As a consequence, the only relevant independent parameters are the volume $V$ and the reduced chemical potential $\mu \equiv \beta \tilde{\mu}$, where $\beta=1 / k_{B} T$ and $\tilde{\mu}$ is the chemical potential of the particle reservoir. The value of $\tilde{\mu}$-the average energy change in the system when an additional particle is introduced-controls the average number of particles inside the volume. Since the system is athermal, to simplify the nomenclature, from now on we will refer to $\mu$ as simply "the chemical potential." In the grandcanonical Monte Carlo simulation performed here, three kinds of trial movements are allowed: displacement, insertion, and deletion of particles. ${ }^{7}$ During each simulation the chemical potential $\mu$ is kept fixed, while the number of particles inside $V$ fluctuates. The simulations are done in two stages. First various short runs are performed to locate the critical region. Then a single long run at the value of $\mu$ close to the putative critical chemical potential $\mu_{c}$ is carried out. From the time series data of the long run, using histogram reweighting techniques, ${ }^{8-10}$ the relevant thermodynamic observables are accurately evaluated throughout the critical region. Another set of measurements at different values of $\mu$ is also performed to check the reliability of the extrapolation. The diffusion trial movements are performed explicitly to help achieve higher densities and to avoid entrapment of the system in low density metastable states.

The lattice gases with hard core exclusion present a transition from a low density fluidlike phase, in which all sites have the same occupational probability, to a large density crystal-like phase in which the system is no longer translationally invariant. Thus, the lattice may be conveniently divided into sublattices which in the high density phase are differently populated. Later we describe the exclusion shells considered in our simulations and the corresponding sublattices.

For the system sizes considered in our simulations, the density $\rho$ and the compressibility $\kappa=L^{2}\left(\left\langle\rho^{2}\right\rangle-\langle\rho\rangle^{2}\right) / \rho^{2}$, where $\langle\ldots\rangle$ is the ensemble average, are not very sensitive to the phase transition. To obtain a quantitative information we, therefore, build order parameters which become nonzero as a system passes from a fluid to an ordered phase. When the transition is continuous, the order parameter $q$ (to be defined later) obeys the usual finite size scaling relation

$$
q=L^{-\beta / \nu} f\left[\left(\mu-\mu_{c}\right) L^{1 / \nu}\right]
$$

where $f$ is a scaling function and $\mu_{c}$ is the critical value of the chemical potential in the thermodynamical limit, $L \rightarrow \infty$. The susceptibility $\chi$ measures the fluctuations of $q$,

$$
\chi=L^{2}\left(\left\langle q^{2}\right\rangle-\langle q\rangle^{2}\right),
$$

and has the scaling
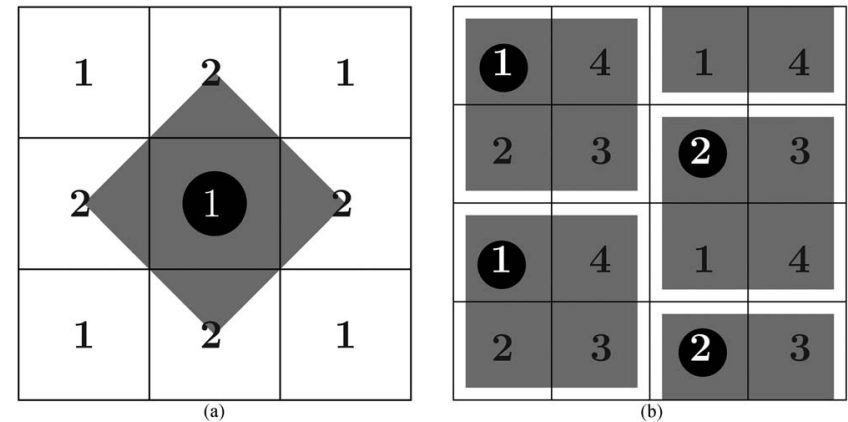

FIG. 1. Lattice division for the (a) $1 \mathrm{NN}$ and (b) $2 \mathrm{NN}$ exclusions: two and four sublattices are needed, respectively. The $1 \mathrm{NN}$ case is equivalent to tilted, nonoverlapping hard squares of length $\lambda=\sqrt{2}$, while in the $2 \mathrm{NN}$ case the squares are not tilted and have $\lambda=2$. The equivalent hard squares are shown in gray.

$$
\chi=L^{\gamma / \nu} g\left[\left(\mu-\mu_{c}\right) L^{1 / \nu}\right],
$$

where $g$ is another scaling function. The exponents $\beta, \gamma$, and $\nu$ are related with the critical behavior of the order parameter, susceptibility, and the correlation length, respectively, and obey the hyperscaling relation $2 \beta / \nu+\gamma / \nu=d$. In the simulations later, one way to obtain $\nu$ is from the behavior of $\partial \ln q / \partial \mu$, whose scaling, from Eq. (1) follows:

$$
\frac{\partial \ln q}{\partial \mu}=L^{1 / \nu} h\left[\left(\mu-\mu_{c}\right) L^{1 / \nu}\right],
$$

where $h(x)=f^{\prime}(x) / f(x)$. An analogous equation is obeyed if we change $q$ by $\chi$. Alternatively, $\nu$ can also be obtained from the behavior of the shifted location of the maxima of $\chi$ and $\kappa$ which approach the thermodynamic limit with $L^{-1 / \nu}$ corrections. Once $\nu$ is known, $\beta$ and $\gamma$ are obtained from Eqs. (1) and (3). There are several methods that can be used to distinguish first order from continuous transitions in numerical simulations, with different levels of success depending on the strength of the transition. For example, the behavior of the many thermodynamical functions (both the average value and the full distribution), the Binder cumulant, etc. In this work, the main signature of a first order transition will be the scaling of thermodynamical variables with the volume of the system, ${ }^{82} L^{2}$.

\section{A. Nearest neighbor exclusion (1NN)}

The simplest nontrivial lattice gas consists of particles which preclude nearest neighbor sites from being occupied. As shown in Fig. 1(a), the lattice may be subdivided into two sublattices, each site being surrounded by the sites of the other sublattice. This system, which can also be interpreted either as $45^{\circ}$ tilted hard squares of linear size $\lambda=\sqrt{2}$ or as hard disks of radius $\sqrt{2} / 2$, has been extensively studied and here we present some results for the sake of both completeness and comparison. Many different approaches have been used to describe its properties on a square lattice: series expansions, ${ }^{3,11-14}$ cluster variational and transfer matrix methods, ${ }^{11,15-24}$ renormalization group, ${ }^{25,26}$ Monte Carlo simulations, ${ }^{27-34}$ Bethe lattice, ${ }^{11,35-39}$ and more recently density functional theory. ${ }^{40}$ Moreover, this model has also been considered because of its interesting mathematical ${ }^{41-43}$ and 
dynamical ${ }^{44-54}$ properties. These studies indicate that the system undergoes a continuous phase transition belonging to the Ising universality class, at $\mu_{c} \simeq 1.33$, when the concentration of particles is close to $\rho=N / L^{2} \simeq 0.37$. Note that the maximum density is $1 / 2$. In higher dimension ${ }^{25,31,32,55-59}$ and other geometries (see Ref. 41, and references therein), the same kind of transition is observed, also belonging to the Ising universality class.

The transition is from the disordered fluid phase, where both sublattices are equally occupied, to a high density ordered phase with most of the particles confined to one sublattice [see Fig. 1(a)]. The order parameter for this system is

$$
q_{1}=\frac{1}{\rho_{\mathrm{MAX}}}\left\langle\left|\rho_{1}-\rho_{2}\right|\right\rangle,
$$

where

$$
\rho_{i}=\frac{1}{L^{2}} \sum_{j \in \mathcal{C}_{i}} \sigma_{j}, \quad i=1,2,
$$

is the density in the $i$ th sublattice $\mathcal{C}_{i}$ (the index $j$ running over all sites in that sublattice), $\rho_{\mathrm{MAX}}=1 / 2$ is the maximum equilibrium density. The variable $\sigma_{j}$ is 0 if the site $j$ is empty and 1 if it is occupied. In the disordered low density regime $\rho_{1}$ $=\rho_{2}$ and $q_{1}=0$ in the thermodynamic limit. At higher densities, one sublattice becomes preferentially occupied, resulting in a finite value of the order parameter. For finite systems the particles switch from one sublattice to the other, analogous to the switching of the magnetization sign in the Ising ferromagnet. ${ }^{60}$ The phase transition appears only in the thermodynamic limit.

\section{B. Nearest and next-nearest neighbors exclusion (2NN)}

Extending the range of interaction, we now consider a lattice gas such that each particle prevents both its nearest and next-nearest neighbors $(2 \mathrm{NN})$ from being occupied. Thus, we define four different sublattices labeled from 1 to 4 as shown in Fig. 1(b). It should be noted that this system is equivalent to hard squares with linear size equal to two lattice spaces $(\lambda=2)$, as is demonstrated in Fig. 1(b); if a particle occupies a position on one sublattice, say 1, the companion sites from sublattices 2 to 4 will be empty. Thus, the maximum possible density, corresponding to a full lattice occupation, is $\rho_{\mathrm{MAX}}=1 / 4$. Although this system has been considered many times in the last decades, ${ }^{6,11,19,22,27,38,51,61-72}$ sometimes as the zero temperature limit of a soft-core potential, ${ }^{22,27,67,72}$ there is still uncertainty of its universality class. The order, location, and even the existence of the transition depend on the approximation method used to study the model. In Table I we show the density, the chemical potential, and the order of the transition obtained by several different methods. Little is known about its critical exponents, Ref. 22.

In the low density regime, the system is fluidlike, without any long ranged order. As the density increases, there is a transition from the disordered phase to a columnar phase, ${ }^{6,11,22,62,66,69}$ where half-filled columns (rows) intercalate with empty ones: the system is ordered along one direc-
TABLE I. Chemical potential and the density at the order-disorder transition along with the technique used and the putative order of the transition for a lattice gas with nearest and next-nearest neighbors. The symbol "?" is used when some uncertainty is acknowledged by the authors. The several techniques used are: transfer matrix (TM), cluster variational method (CVM), low and high series expansion, generalizations of the Bethe method, interface method (Ref. 75), density functional theory (DFT), and Monte Carlo. Notice the wide range of values found for the critical chemical potential.

\begin{tabular}{llccc}
\hline \hline \multicolumn{1}{c}{$\mu_{c}$} & $\rho_{c}$ & Method & Order & References \\
\hline 4.7 & 0.24 & TM & Cont $(?)$ & 22 and 70 \\
3.115 & 0.225 & CVM & & 66 \\
3.889 & 0.222 & CVM & & 69 \\
5.3 & 0.238 & TM & Cont & 62 \\
- & - & Series & No & 11 and 64 \\
4 & 0.23 & TM & $(?)$ & 11 \\
- & - & TM & No & 64 \\
2.846 & 0.202 & Bethe & Cont & 11 \\
4.91 & - & Interface & Cont & 67 \\
& & Bethe & First & 38 \\
2.406 & 0.191 & DFT & Cont & 6 \\
4.574 & 0.233 & Monte Carlo & Cont & This work \\
\hline \hline
\end{tabular}

tion but fluid along the other. Since there is no interaction between the particles (apart the exclusion), there is no alignment between particles in the neighboring columns (rows), that is, there is no solid phase. ${ }^{73}$ As a consequence, the close packed configuration is not unique-each column (or row, but not both) may be shifted by one lattice space thus introducing an $\mathcal{O}(L)$ contribution to the entropy at this maximum density. Interestingly, no transition was found for the analogous $d=3$ system of hard cubes with $\lambda=2$, (Ref. 59) on a cubic lattice. On the other hand, nearest and next-nearest neighbors exclusion in $d=3$ presents a weak first order transition. ${ }^{59}$ Other lattices have also been considered, see Refs. 41 and 74, and references therein.

The order parameter needed to study this transition is a generalization of the $1 \mathrm{NN}$ Eq. (5),

$$
q_{2}=\frac{1}{\rho_{\mathrm{MAX}}}\left\langle\left|\rho_{1}-\rho_{3}\right|+\left|\rho_{2}-\rho_{4}\right|\right\rangle .
$$

In the fluid phase all the sublattices are equally occupied and $q_{2}=0$. The order parameter becomes nonzero when the symmetry between the sublattices is broken. For the columnar phase two sublattices are preferentially occupied, while the remaining two have a much smaller density. For example, particles may be located on sublattices 1 and 2 [as in Fig. 1(b)], while sublattices 3 and 4 are almost empty, etc.

\section{First, second, and third neighbor exclusion (3NN)}

The third nearest neighbor (3NN) case has its range of exclusion extended up to third nearest neighbors. As shown in Fig. 2, this can be interpreted both as hard-core pentamers ${ }^{76}$ or slightly tilted squares with $\lambda=\sqrt{5}$. Previous studies of this system were based on series expansions, ${ }^{11,77}$ cluster variational and transfer matrix, ${ }^{11,19,20,77-80}$ Bethe method, ${ }^{11}$ etc. It is now known that at high densities, the system undergoes a first order transition to a doubly degenerated ordered phase, ${ }^{81}$ the ground state configurations being related by chirality, see Figs. 2(b) and 2(c). This is reflected 

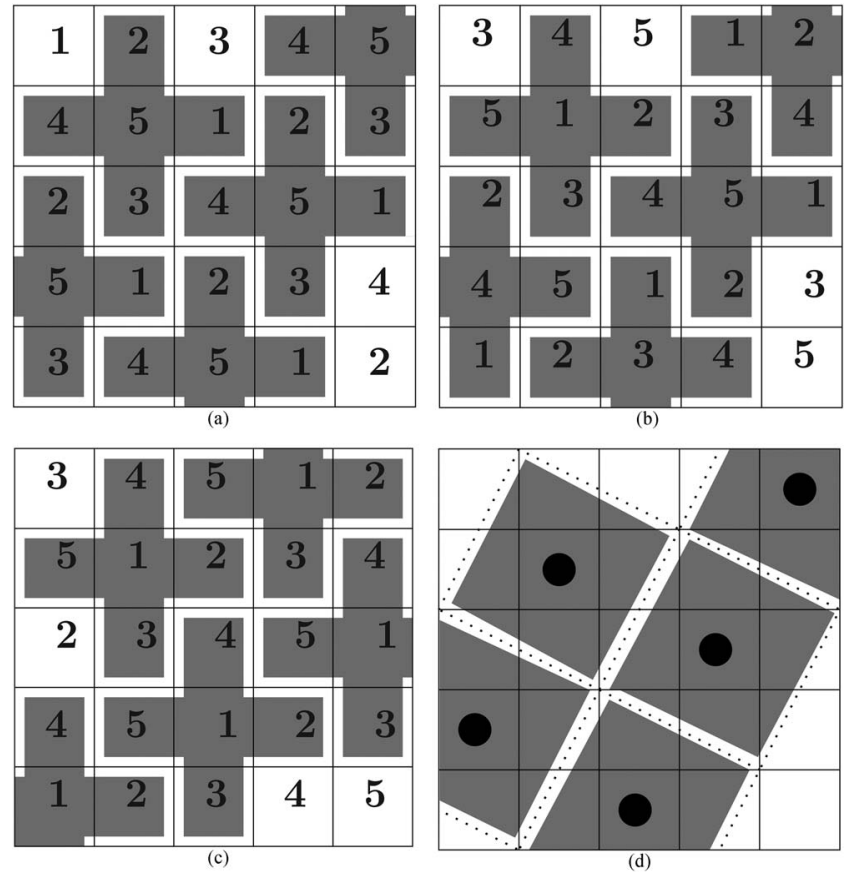

FIG. 2. Two possible labelings for the sublattices in the 3NN case-panels (a) and (b). For the same high density configuration in (a) all particles are on the same sublattice (five in this example), while in (b) all sublattices are equally populated. For the labeling (b), panel (c) shows another configuration in which particles occupy only one sublattice. Note that the ground states shown in panels (b) and (c) are chiral, in the sense that the two are the reflections of one another about the left-to-right body diagonal. The exclusion problem can be formulated either in terms of the symmetric crossshaped pentamers (shown in gray) or the tilted hard-squares of side length $\lambda=\sqrt{5}(\mathrm{~d})$

in the two possible ways of introducing sublattices to describe the symmetry breaking: for each of the possible enantiomorph ground states, there is a corresponding labeling (called A and B) such that all particles belong to only one sublattice [see, for example, Figs. 2(a) and 2(b)].

For a given configuration, since we do not know a priori which of the two labelings is more relevant, we measure the following quantity for each of them:

$$
q_{\mathrm{A}}=\sum_{i=1}^{5} \sum_{j>i}^{5}\left|\rho_{i}^{\mathrm{A}}-\rho_{j}^{\mathrm{A}}\right|
$$

and equivalently for the labeling B. Note that in these sums the density of each sublattice appears four times. $\rho_{i}$ is given by Eq. (6) for the chosen labeling. This quantity is zero for both labelings in the fluid phase, where all sublattices are equally populated, while it becomes nonzero above the critical density (for one of the labelings). The corresponding order parameter is the difference of the two measures

$$
q_{3}=\frac{1}{4 \rho_{\mathrm{MAX}}}\left\langle\left|q_{\mathrm{A}}-q_{\mathrm{B}}\right|\right\rangle
$$

Again, this order parameter is a natural extension of $q_{2}$. At the close packed configuration, where the density is $\rho_{\mathrm{MAX}}$ $=1 / 5, q_{\mathrm{A}}=4 \rho_{\mathrm{MAX}}$, and $q_{\mathrm{B}}=0$ (or vice-versa): the first labeling is such that all particles sit on the same sublattice, while in the second labeling all sublattices have the same density. In both cases, however, $q_{3}=1$.
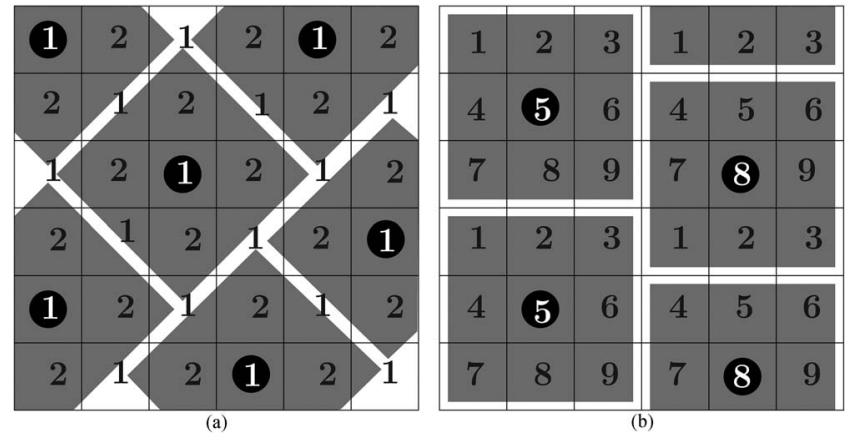

FIG. 3. Sublattice division for the exclusion of up to (a) $4 \mathrm{NN}$ and (b) $5 \mathrm{NN}$. These are equivalent to hard squares with $\lambda=2 \sqrt{2}$ and 3 , respectively. Notice that in both cases the ordered phase is columnar (as in the $2 \mathrm{NN}$ case), but in the former, the columns are along the diagonals.

\section{D. $4 \mathrm{NN}$}

The subsequent situation considers exclusion of up to four nearest neighbor sites (4NN). As seen in Fig. 3(a), this is equivalent to tilted hard squares of side length $\lambda=2 \sqrt{2}$. Nisbet and Farquhar ${ }^{79}$ studied this model using transfer matrices and concluded that the transition is either continuous or very weak first order. The close packed configuration shown in Fig. 3(a) is similar to the 1NN case, although less dense (the close packed density is $\rho_{\mathrm{MAX}}=1 / 8$ ), but it is not unique: analogously to the $2 \mathrm{NN}$ case, the columns (which in this case are tilted), are independent and may slide along the diagonals. This does not change, however, the sublattice that the particles occupy. Notice also that this does not occur in the tilted diagonals of the $1 \mathrm{NN}$ and $3 \mathrm{NN}$ cases, the squares being always aligned. Thus, the order parameter should be insensitive to a diagonal displacement of a whole (tilted) column and it is enough to subdivide the lattice in only two sublattices, in analogy with the $1 \mathrm{NN}$ case. Indeed, the transition to the ordered state may be analyzed with the same order parameter, Eq. (5).

\section{E. $5 \mathrm{NN}(\lambda=3)$}

Finally, we considered a lattice gas composed of hard squares with linear size equal to three lattice spaces, $\lambda=3$. In terms of exclusion shells, this is also equivalent to excluding up to the fifth nearest neighbors, as shown in Fig. 3(b). The close packed configuration, like in the $2 \mathrm{NN}(\lambda=2)$ case, is not uniquely defined and has a density $\rho_{\mathrm{MAX}}=1 / 9$. In $d=3$, this system undergoes a weak first order phase transition as density increases, ${ }^{59}$ although there seems to be no information concerning the $d=2$ case. The corresponding sublattice division is analogous to the $2 \mathrm{NN}$ case, only that now nine sublattices are necessary, the order parameter being

$$
\begin{aligned}
q_{5}= & \frac{1}{2 \rho_{\mathrm{MAX}}}\left\langle\left|\rho_{1}-\rho_{5}\right|+\left|\rho_{1}-\rho_{9}\right|+\left|\rho_{5}-\rho_{9}\right|+\left|\rho_{2}-\rho_{6}\right|\right. \\
& +\left|\rho_{2}-\rho_{7}\right|+\left|\rho_{6}-\rho_{7}\right|+\left|\rho_{3}-\rho_{4}\right|+\left|\rho_{3}-\rho_{8}\right| \\
& \left.+\left|\rho_{4}-\rho_{8}\right|\right\rangle .
\end{aligned}
$$

Equation (10) uses the labeling of sublattices depicted in Fig. 3(b). 


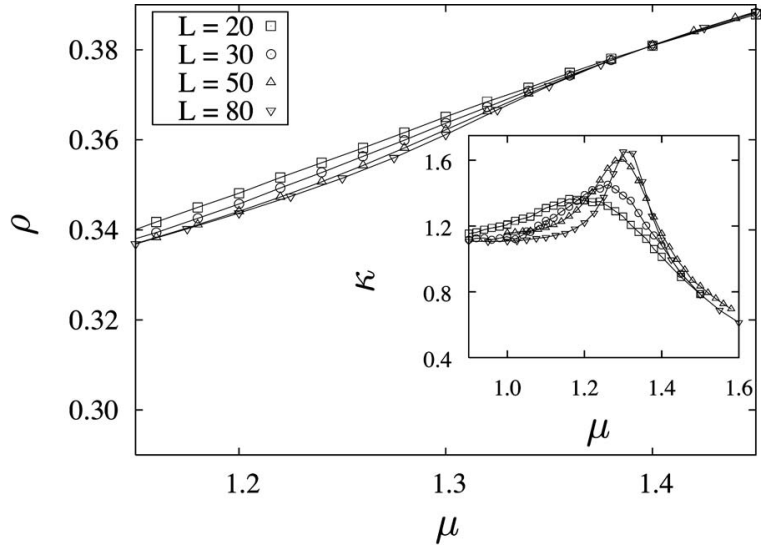

FIG. 4. Density $\rho$ as a function of the chemical potential $\mu$ for the $1 \mathrm{NN}$ exclusion for several lattice sizes. The inflection close to the transition point $\left(\mu_{c} \simeq 1.33\right)$ is very small, being noticeable only for larger system sizes. Inset: compressibility $\kappa$ as a function of the chemical potential.

\section{RESULTS}

\section{A. $1 \mathrm{NN}$}

In the case of nearest neighbor exclusion, the change in density at the transition is quite subtle, the inflection point being only noticeable for the largest sizes simulated, as seen in Fig. 4. The transition is more clearly seen in the plot of the 1NN order parameter, Eq. (5), as shown in Fig. 5 for increasing lattice sizes: $q_{1}$ changes from a small value (fluid phase) to a value close to unity (checkerboard pattern). The behavior of $q_{1}$ is characteristic of a second order phase transition and indeed, all curves collapse onto a universal curve $f_{1}$, as shown in the inset of Fig. 5, whose scaling obeys Eq. (1). In accordance with the previous studies, ${ }^{3,47}$ this transition occurs at $\mu_{c} \simeq 1.33$, corresponding to $\rho_{c} \simeq 0.37$, and belongs to the universality class of the two-dimensional Ising model, with exponents $\nu=1$ and $\beta=1 / 8$.

Another quantity of interest, shown in Fig. 6, is the staggered susceptibility $\chi_{1}=L^{2}\left(\left\langle q_{1}^{2}\right\rangle-\left\langle q_{1}\right\rangle^{2}\right)$ which measures the order parameter fluctuations. As the system size increases, the susceptibility peak becomes higher and narrower, shifting to larger values of the chemical potential. All curves can be

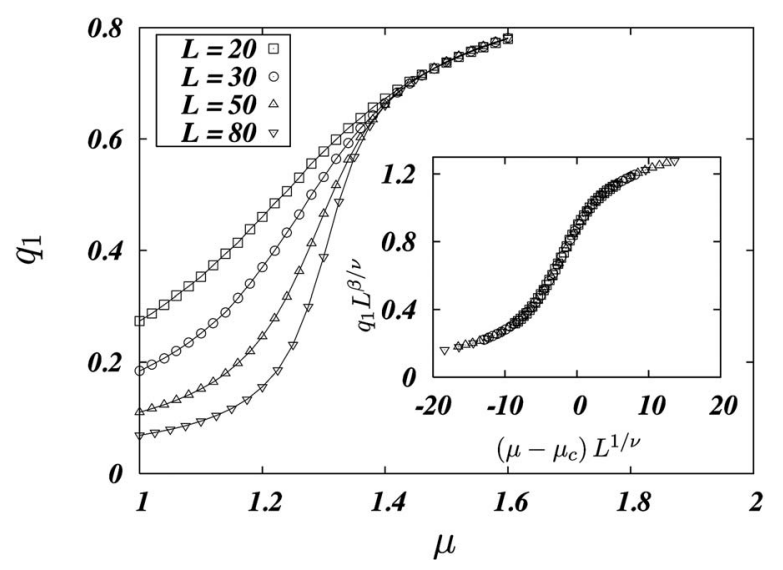

FIG. 5. Order parameter $q_{1}$, Eq. (5), as a function of the chemical potential $\mu$, for the $1 \mathrm{NN}$ exclusion for several lattice sizes, along with the corresponding data collapse (inset). The critical exponents used are those of the two dimensional Ising model, $\nu=1$ and $\beta=1 / 8$.

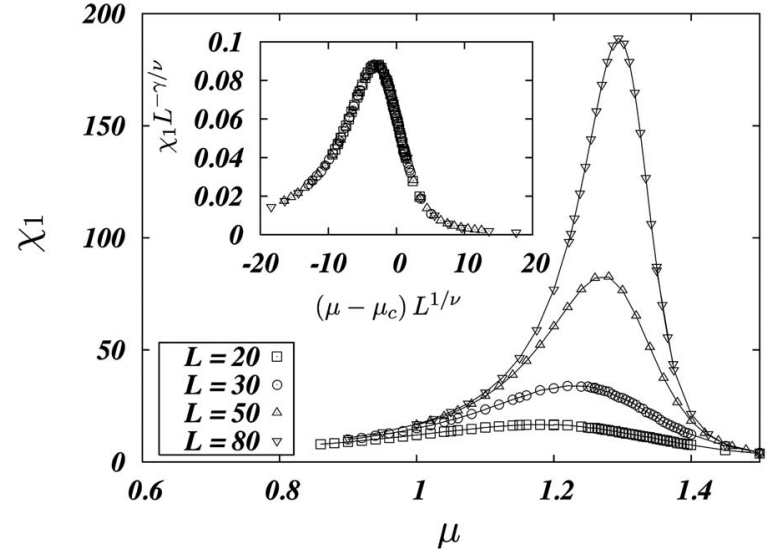

FIG. 6. Order parameter fluctuations $\chi_{1}$, Eq. (2), as a function of the chemical potential $\mu$ for the $1 \mathrm{NN}$ model using several lattice sizes. In the inset, the corresponding data collapse is shown using the critical exponents of the bidimensional Ising model, $\gamma=7 / 4$ and $\nu=1$.

collapsed onto a universal curve whose scaling obeys Eq. (3), as shown in the inset of Fig. 6. Again, the exponents are those of the two-dimensional Ising model, $\gamma=7 / 4$ and $\nu=1$. Much less clear are the fluctuations in density, that is, the compressibility $\kappa$, as shown in the inset of Fig. 4. Although the peak grows with $L$, the sizes considered are still too small to try to obtain useful information from the data collapse. Notice that in this case, the maximum is expected to increase with the logarithm of $L(\alpha=0)$.

\section{B. $2 \mathrm{NN}$}

Analogously to the previous case, when the range of exclusion increases to include the next-nearest neighbors, it is very hard to see the transition by only looking at the behavior of the density as a function of the chemical potential. In the critical region only for larger system sizes the inflection point becomes noticeable, as is shown in Fig. 7 and the change in density is roughly one order of magnitude smaller as compared with the 1NN case. On the other hand, the order parameter $q_{2}$, Eq. (7), shows a fast increase between $\mu=4$ and 5, Fig. 8, as the system enters the ordered (columnar)

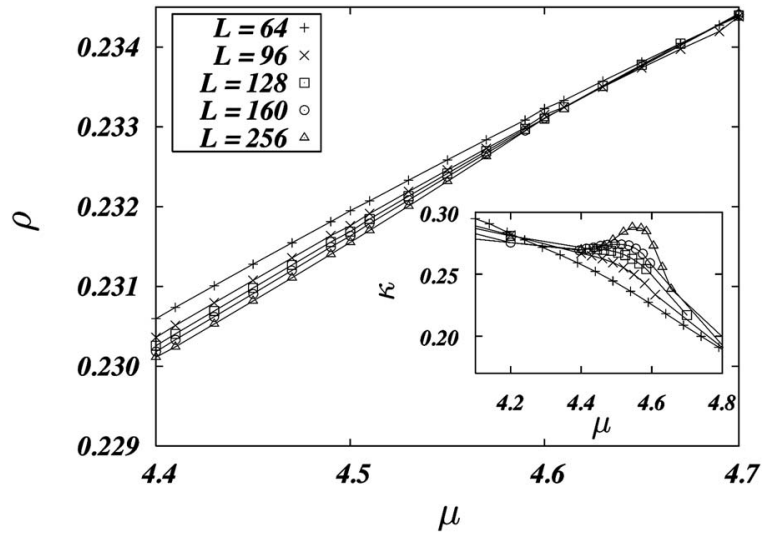

FIG. 7. Density as a function of chemical potential for several lattice sizes for the gas of $2 \mathrm{NN}$ exclusion region where the inflection point appears (noticeable only for the larger system sizes). Inset: compressibility $\kappa$ in the region where the transition is found. Only for the largest lattice simulated the maximum is prominent. 


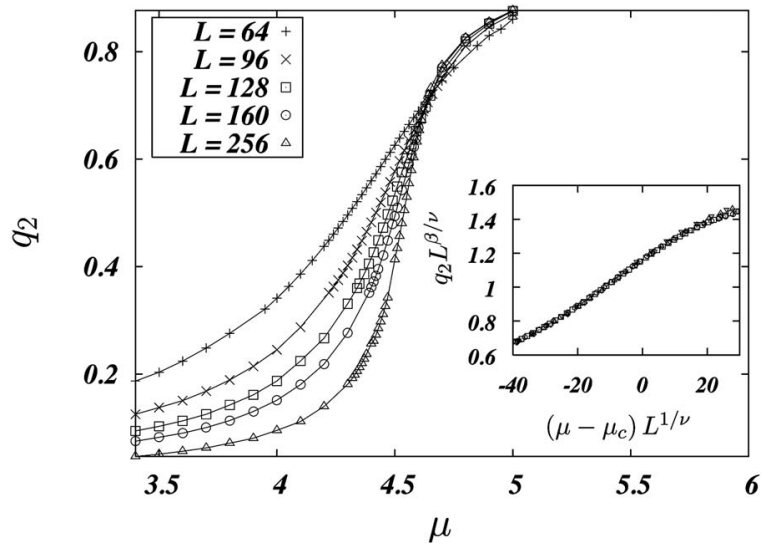

FIG. 8. Order parameter for $2 \mathrm{NN}$ [Eq. (7)] as a function of the chemical potential for different lattice sizes. Small values of $q_{2}$ signal that the system is disordered. For $q_{2}$ closer to unity, the system is in a columnar phase. Inset: data collapse onto a universal curve for the larger system sizes $L$ $=128,160,256,320$, and 360. A very good collapse is obtained for $\mu_{c}$ $=4.574, \nu=0.94$, and $\beta / \nu=0.125$, in close agreement with the exact Ising values. The collapse remains good if we use the Ising value $\nu=1$.

phase from the disordered (fluid) one. The the existence, location, order, and exponents of this transition have remained elusive, as can be seen from a very broad range of approximate values presented in Table I and the lack of any estimates for the critical exponents. We find that neither the density nor the order parameter $q_{2}$ present any signs of discontinuity. Within the range of lattice sizes considered no hysteresis, discontinuities, nor any evidences of the coexistence in the density and or the order parameter histograms has been observed. We conclude that the transition is continuous, in agreement with most of the previous studies.

Indeed, the $q_{2}$ data can be collapsed onto a universal curve [as in Eq. (1)], with $\mu_{c} \simeq 4.574, \beta / \nu=0.125$, and $\nu$ $=0.94$. Remarkably, $\beta / \nu$ has the same value of the (exact) Ising model, $1 / 8=0.125$. Although $\nu$ is a little bit smaller than the Ising value, a good collapse is still obtained with $\nu=1$ and, as we will see later, is in agreement with other measures of this exponent. The density, measured for several systems sizes at $\mu_{c}$, or at the peak of the susceptibility extrapolate when $L \rightarrow \infty$ to $\rho_{c}=0.233$. The value of $\mu_{c}$ is also compatible with the crossing point of the Binder cumulant for the order parameter and with the extrapolated position of the susceptibility, Fig. $9, \mu_{c} \simeq 4.578$.

We also measured the order parameter and the density fluctuations - that is, the staggered susceptibility $\chi_{2}$, Eq. (2), and the compressibility $\kappa_{2}$, respectively, as shown in Figs. 10 and 7 (inset) as a function of the chemical potential. In both cases, as the system size increases the curves get less broad and their height becomes larger. However, the maximum of $\kappa_{2}$ only becomes noticeable for the larger simulated lattices and no reliable information can be obtained from its scaling, within the range of $L$ considered here. The shift of the position of the peak of $\chi_{2}$ from $\mu_{c}$ behaves as $L^{-1 / \nu}$ [in the same way as the position of the maximum in Eq. (4)], from where $\nu$ can be estimated, as shown in Fig. 9. In addition, the height of the peak of Eq. (4) increases with $L^{1 / \nu}$. The exponent $\nu$ evaluated from both measurements is very close to the Ising value, see the caption of Fig. 9 for details. We conjecture,

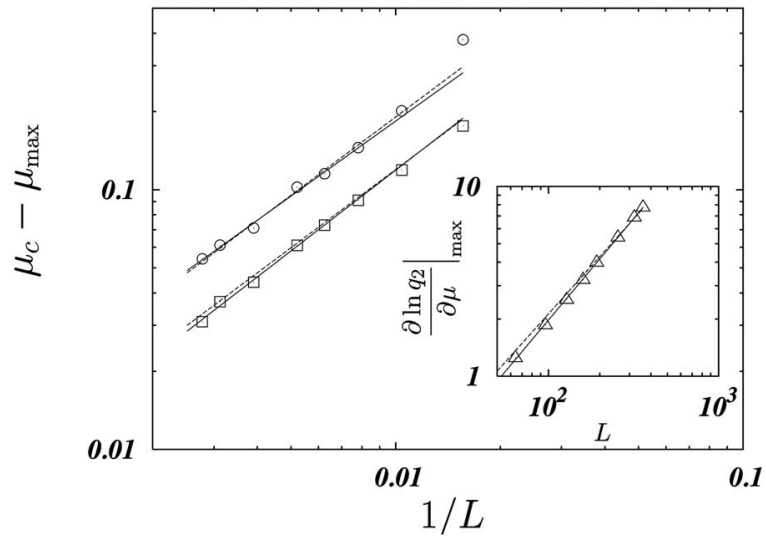

FIG. 9. Several estimates of the exponent $\nu(2 \mathrm{NN})$ : the position of the maxima of Eq. (4) (top curve) and the susceptibility $\chi_{2}$ (bottom), that shift as $L^{-1 / \nu}$, as a function of $L$. The solid lines are power-law fits neglecting the smaller sizes, from which we get $\nu \simeq 1.042$ and $\nu \simeq 0.968$, top and bottom, respectively. These values are very close to the Ising $\nu=1$, as can be seen by the fits fixing the exponent to this value (dashed lines). Also from the fit of the location of the maximum of the susceptibility we have an independent estimate of the transition: $\mu_{c} \simeq 4.578$. Inset: the height of the maximum of Eq. (4), increasing as $L^{1 / \nu}$, as a function of $L$. Again the dashed line is a fit with $\nu=1$, while the solid line is the best fit with $\nu \simeq 0.937$.

therefore, that the exact value is indeed $\nu=1$. The height of the peak of $\chi_{2}$ increases as $L^{\gamma / \nu}$, and the fit yields $\gamma / \nu$ $=1.755$ (bottom inset of Fig. 10), giving an excellent data collapse as can be seen in the top inset of Fig. 10. Again, this value is very close to the known exact value for the Ising model, $\gamma=7 / 4=1.75$. The fact that the lattice gas of $2 \mathrm{NN}$ exclusion is in the same universality class as the Ising model is quite remarkable considering that the symmetries of the ground state and of the order parameter are so very different from those of $1 \mathrm{NN}$ model.

\section{C. $3 \mathrm{NN}$}

The lattice gas with exclusion of up to $3 \mathrm{NN}$ is important since it presents, differently from the previous cases, a first order transition, showing that the nature of the transition depends on the range of exclusion, as was observed previously. Recently, Eisenberg and Baram ${ }^{80}$ precisely located the tran-

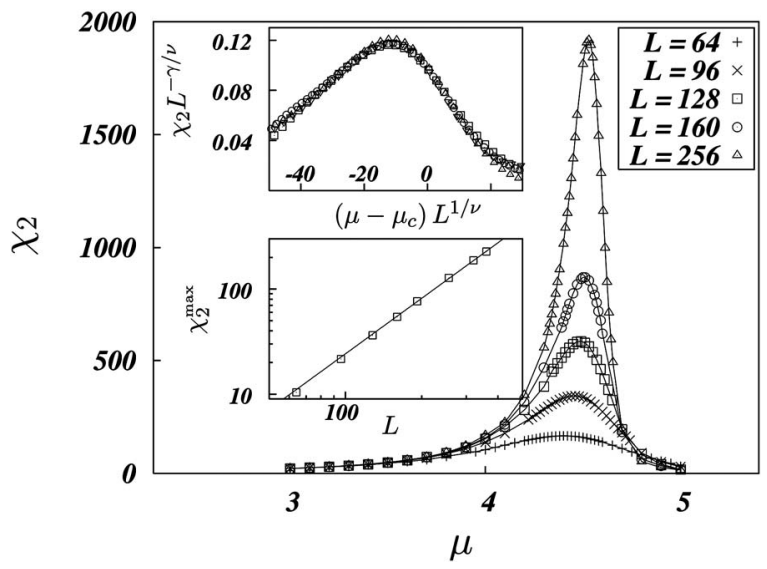

FIG. 10. The staggered susceptibility $\chi_{2}$ as a function of $\mu$ for several lattice sizes in the $2 \mathrm{NN}$ case. Inset: data collapse (top) onto a universal curve with exponent $\gamma$ obtained from fitting the height of the maximum of $\chi_{2}$ as a function of $L$ (bottom), $\gamma / \nu=1.755$, along with $\nu=1$. 


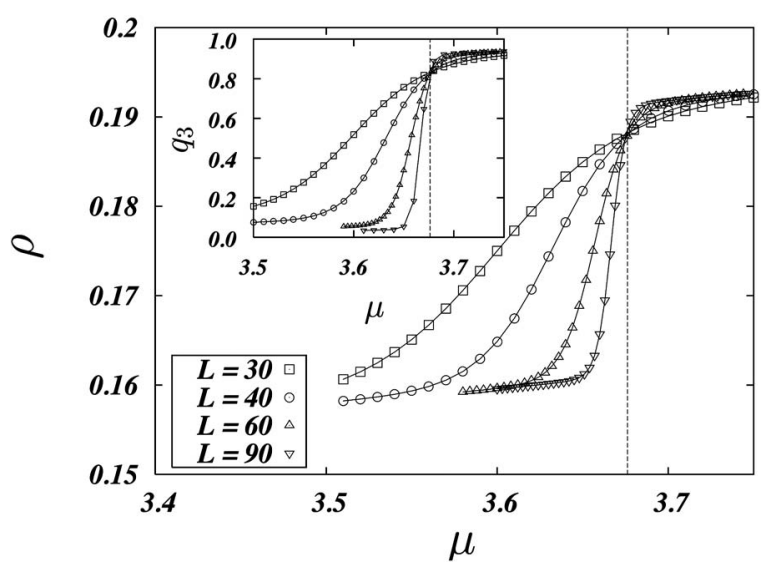

FIG. 11. Density as a function of chemical potential for the $3 \mathrm{NN}$ exclusion model. At low densities the system behaves as a fluid and undergoes a first order phase transition to an ordered phase as the density increases. The vertical dashed line at $\mu=3.6762$ is the infinite size extrapolation obtained with matrix methods (Ref. 80) and agrees well with the crossing point of the curves. Inset: the order parameter as a function of the chemical potential.

sition, using matrix methods, obtaining $\mu_{c} \simeq 3.6762$. In Fig. 11 and its inset, both the density and the order parameter, respectively, show a rapid rise as the chemical potential increases, passing from a fluid, low density phase, to an ordered, high density phase. Notice also the good agreement between the matrix prediction for the transition and our Monte Carlo data. The first order character of this transition is clearly seen in the behavior of the compressibility ${ }^{77}$ and the fluctuations of the order parameter, shown in Figs. 12 and 13. All the relevant scaling is with the volume of the system $L^{2}(\nu=1 / d),{ }^{82}$ as can be seen in the inset of both figures. Excellent data collapses were obtained with $\mu_{c}=3.6746$, very close to the value calculated using the transfer matrix. ${ }^{80}$ This value is also very close to the ones obtained by extrapolating the positions of the compressibility and the susceptibility peaks as a function of the system size and with the crossing points of the density and the order parameter for the two largest system sizes.

\section{4NN}

When excluding up to four neighbors, the close packed state resembles both the $1 \mathrm{NN}$ and $2 \mathrm{NN}$ case. On one hand,

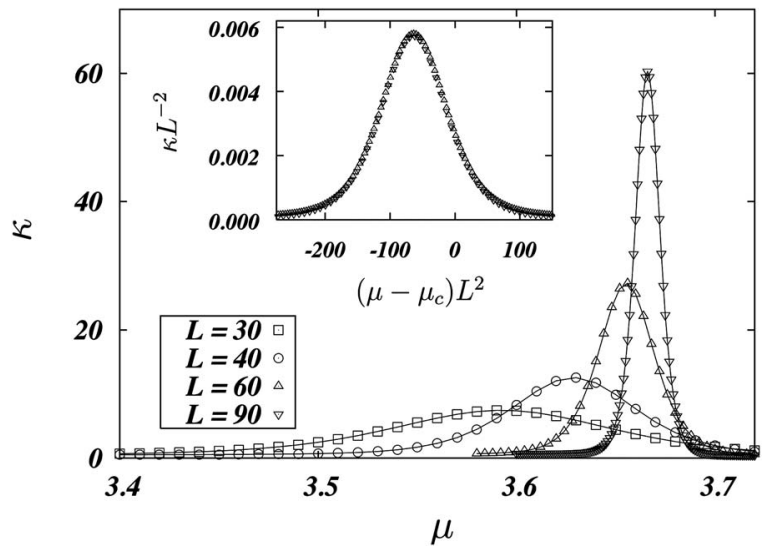

FIG. 12. Compressibility $\kappa$ as a function of the chemical potential for the $3 \mathrm{NN}$ exclusion for several lattice sizes. Inset: after rescaling the height and the width of the curves by $L^{2}$ and $L^{-2}$, respectively, an excellent collapse is observed for the two largest systems with $\mu_{c}=3.6746$.

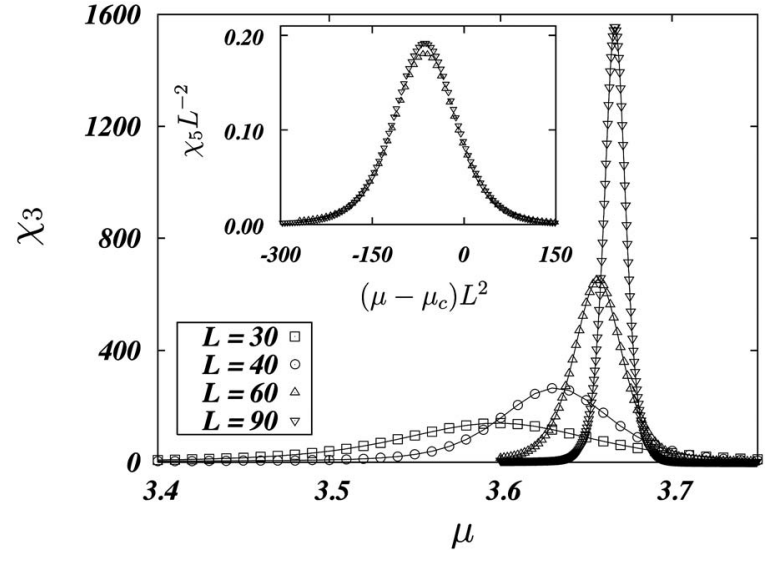

FIG. 13. Staggered susceptibility $\chi_{3}$ as a function of the chemical potential for the $3 \mathrm{NN}$ exclusion case for several lattice sizes. Inset: after rescaling the height and the width of the curves by $L^{2}$ and $L^{-2}$, respectively, an excellent collapse is observed with $\mu_{c}=3.6746$.

particles are located on one of the two sublattices, arranged along the diagonals of the system (although skipping one every two sites); on the other hand, these diagonals are independent "columns" that may slide, originating a $2^{L}$-degenerated ground state with density $1 / 8$. Surprisingly, within the size range available, the transition appears to be continuous and in the Ising universality class, contradicting the Landau-Lifshitz theory which predicts that this transition should be of first order. ${ }^{65}$

As in the $2 \mathrm{NN}$ case, several estimates of $\nu$ can be obtained. From the positions of the maxima of Eq. (4) and $\chi_{1}$ we obtain, Fig. $14, \nu \simeq 0.923$ and $\nu \simeq 0.999$, respectively. The last one is more reliable since the peaks of $\chi_{1}$ are less broad, thus allowing a more precise location of the maxima. A further measure of $\nu$ is obtained from the height of Eq. (4) and gives $\nu \simeq 1.029$. As in the $2 \mathrm{NN}$ case, these values oscillate around the Ising $\nu=1$ and, again, we conjecture that this is

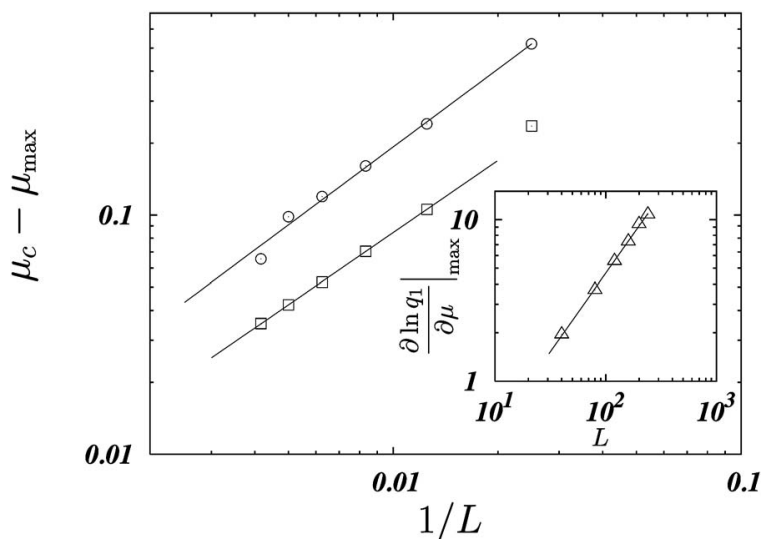

FIG. 14. Several estimates of the exponent $\nu$ (4NN): the position of the maxima of Eq. (4) and the susceptibility $\chi_{1}$ (bottom), that shift as $L^{-1 / \nu}$, as a function of $L$. The solid lines are power-law fits neglecting the smaller sizes, from which we get $\nu \simeq 0.923$ and $\nu \simeq 0.999$, top and bottom curves, respectively. These values are indeed very close to the Ising one, $\nu=1$ (notice also that the peaks of Eq. (4) are broader than those of $\chi_{1}$, and their positions are less reliable). Also from the fit of the location of the maximum of the susceptibility we have an independent estimate of the transition point: $\mu_{c}$ $\simeq 4.705$. Inset: the height of the maximum of Eq. (4), increasing as $L^{1 / \nu}$, as a function of $L$. The solid line is a fit with $\nu \simeq 1.029$. 


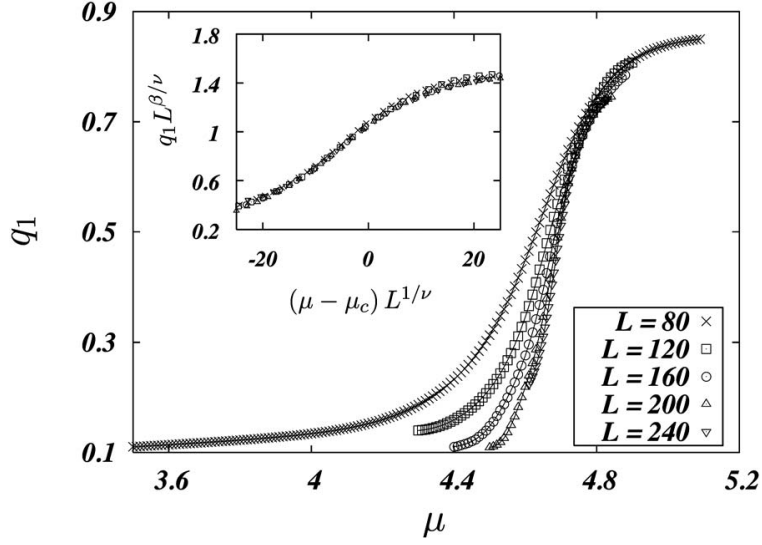

FIG. 15. Order parameter for the 4NN exclusion [Eq. (5)] as a function of the chemical potential for different lattice sizes. For large values of $q_{1}$, the system is in a columnar phase with columns aligned along the diagonals. Inset: collapse of data onto a universal curve with $\nu=1, \beta=0.125$, and $\mu_{c}$ $=4.705$. Equally good collapse is obtained with a broad range of $\beta$.

the exact value. Indeed, this exponent also fits quite well with the data. As before, the transition is not clear from the behavior of the density, while a properly defined order parameter is able to capture it well, Fig. 15. The transition from the ordered, tilted columnar phase, to the fluid phase is found to occur at $\mu_{c} \simeq 4.705$, corresponding to $\rho_{c} \simeq 0.110$.

The order parameter and its collapse are shown in Fig. 15 . The collapse is not very sensitive to the chosen value of $\beta$ and any value in the broad range between 0.12 and 0.14 will do well. We thus present the collapse with $\beta / \nu=0.125$, the Ising value. Although this is rather arbitrary, the closeness of both $\nu$ and $\gamma$ (see later) to the Ising values inspire us to believe that indeed this system is in the Ising universality class.

The fluctuations of the order parameter are shown in Fig. 16. The dependence of the susceptibility peak on the system size is shown in the lower inset, yielding $\gamma / \nu=1.681$. With these values for the exponents, all curves can be collapse onto a universal one. An equally good collapse is obtained if instead of this value of $\gamma$ we use the Ising one, 1.75. We conclude that the phase transition in the lattice gas with 4NN exclusion is in the Ising universality class.

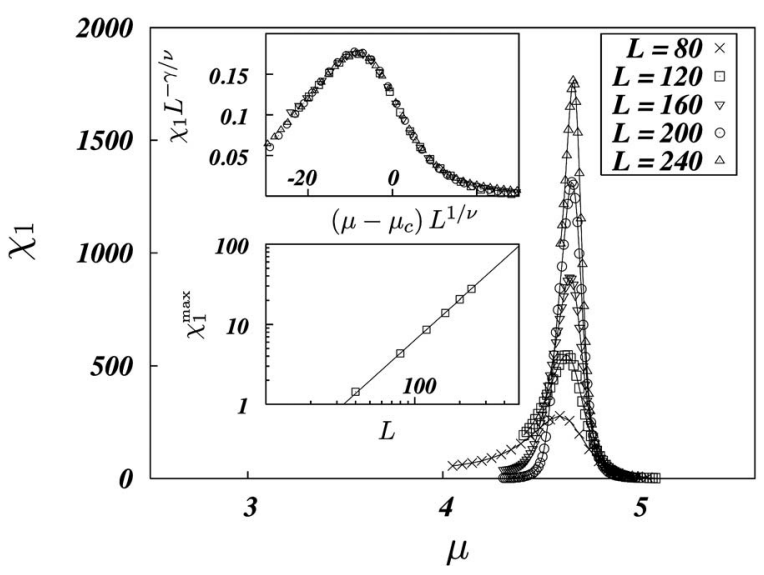

FIG. 16. The staggered susceptibility $\chi_{1}$ as a function of $\mu$ for several lattice sizes for 4NN exclusion. Insets: (top) data collapse onto a universal curve with exponent $\gamma$ obtained from fitting the height of the maximum of $\chi_{1}$ as a function of $L$ shown in the bottom inset; $\gamma / \nu=1.681$ and $\nu=1$.

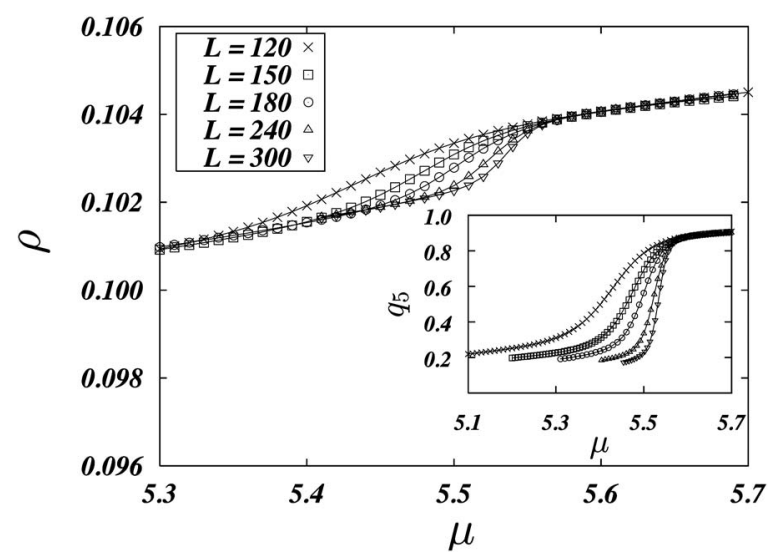

FIG. 17. Density as a function of the chemical potential for $5 \mathrm{NN}$ exclusion $(\lambda=3)$ for different lattice sizes. The steepness increases as the system develops a discontinuity. Inset: order parameter $q_{5}$ as a function of $\mu$ for the same system sizes.

\section{E. $5 \mathrm{NN}(\lambda=3)$}

The lattice gas with exclusion of up to five nearest neighbors is equivalent to hard square particles with side length $\lambda=3$. In this case, as for the $2 \mathrm{NN}$ and $4 \mathrm{NN}$, the system does not have a uniquely defined close packed configuration which can bring problems when approximate (analytical) methods such as series expansions and cluster variational methods are applied.

When the density is measured as a function of the chemical potential, Fig. 17, there is a fast increase between $\mu=5.5$ and 5.6 and the curves become steeper as the system size gets larger signaling the ordering of the system. However, the increase in the density is rather small, while a much more pronounced change is observed for the order parameter, Eq. (10). The fluctuations of the order parameter, shown in Fig. 18, can be well collapsed with a $L^{-2}$ scaling (the volume of the system), at least for the larger sizes considered. This is a signature of a first order transition. The location of the maximum depends on $L$ and obeys the $L^{-2}$ shift with $\mu_{c}$ $\simeq 5.554$. There seem to be, however, strong finite size effects for smaller system sizes, as observed when trying to collapse all the curves-only for the larger sizes considered the col-

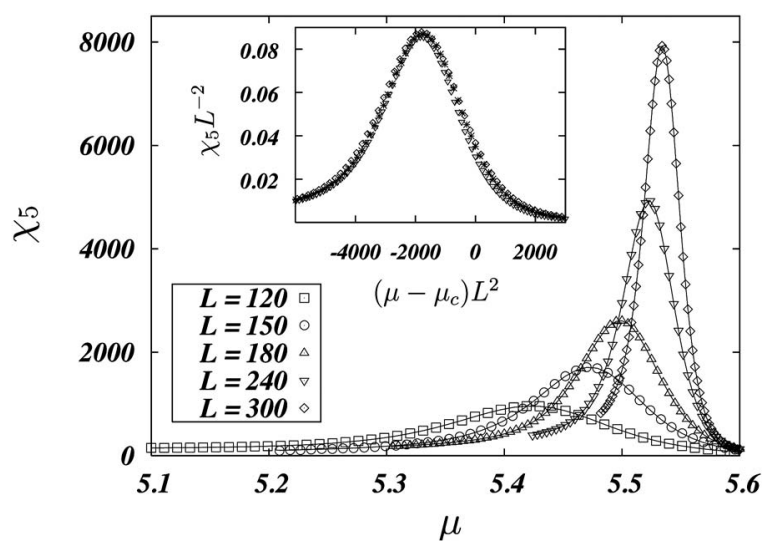

FIG. 18. Staggered susceptibility $\chi_{5}$ as a function of the chemical potential for the $5 \mathrm{NN}$ exclusion $(\lambda=3)$ for several lattice sizes. Inset: after rescaling the height and the width of the curves by $L^{2}$ and $L^{-2}$, respectively, a good collapse is observed for the larger simulated sizes with $\mu_{c}=5.554$. 
lapse is sufficiently good. For the density fluctuations (not shown), even stronger finite size effects are seen. This is probably due to the fact that the increase in the compressibility with $L$ is very small and the nonsingular part of the free energy cannot be discarded when compared to the singular one. This seems to be true for all the cases considered in this paper (being least pronounced for $3 \mathrm{NN}$ exclusion).

\section{CONCLUSIONS}

We used the grand-canonical Monte Carlo simulations to study the order-disorder transition in lattice gases composed of particles with extended hard core on a two dimensional square lattice. In agreement with earlier studies, ${ }^{3}$ the $1 \mathrm{NN}$ exclusion-corresponding to hard squares of length $\sqrt{2}$ tilted by $45^{\circ}$ - was found to undergo a continuous order-disorder transition in the Ising universality class. The lattice gas with exclusions of up to $2 \mathrm{NN}$ was also found to undergo a continuous phase transition in the Ising universality class, while the Landau-Lifshitz (LL) theory ${ }^{65}$ predicts that this transition should be in the universality class of the XY model with cubic anisotropy. The lattice gas with exclusions of up to $3 \mathrm{NN}$ was found to undergo a discontinuous order-disorder transition, in agreement with the earlier transfer matrix calculations ${ }^{80}$ and the LL theory. ${ }^{65}$ On the other hand, the gas of $4 \mathrm{NN}$ exclusions once again exhibited a continuous phase transition in the Ising universality class - a conclusion which contradicts the LL prediction of a first order phase transition. ${ }^{65}$ Finally, the lattice gas with exclusions of up to $5 \mathrm{NN}$ was found to undergo a discontinuous phase transition, in agreement with the LL theory.

The failure of the symmetry based Landau-Lifshitz theory to correctly predict the universality class and the order of the phase transition for some two-dimensional lattice gases is not very surprising. It has been known for a long time that while the Landau-Lifshitz theory works well in three dimensions, its foundations are much shakier in two dimensions (2D). For example, one of its requirements for the existence of a continuous phase transition is the absence of third order invariants of the irreducible representation of the space group of the high symmetry phase to which the order parameter belongs. In 2D this condition is known to breakdown - both three and four state Potts models undergo a continuous phase transition, even though their LandauGinzburg-Wilson (LGW) Hamiltonians possess third order invariants. ${ }^{83,84}$ In view of this problem, Domany et al. have avoided the use of this "Landau rule" in their classification of the continuous order-disorder transitions of 2D lattice gases. ${ }^{65}$ Nevertheless, the fact that one of the Landau rules fails, can already serve as a strong warning against a complete reliance on the symmetry arguments for twodimensional systems. The fundamental difficulty is that $2 \mathrm{D}$ is the lower critical dimension for lattice gases. The fluctuation are very important and can easily modify the order of the phase transitions from the one predicted by mean-field. Furthermore, in 2D one can no longer rely on the renormalization group theory $(\mathrm{RG})$ to justify a simple low order polynomial form of the LGW Hamiltonian. Unlike in three dimensions, where it is possible to show that the high order invariants in the LGW Hamiltonian renormalize to zero under the action of $\mathrm{RG}$, in 2D all the operators are equally relevant-thus invalidating a simple low order polynomial form of the LGW Hamiltonian. In the case of the isotropic $\mathrm{XY}$ model the situation is particularly dire since the symmetry based construction of the LGW Hamiltonian fails to consider the topological excitations which lead to the KosterlitzThouless phase transition in this system. ${ }^{85-87}$ It is, therefore, unlikely that symmetry considerations alone will be sufficient to predict the universality class and the transition order for all 2D lattice gases.

Nevertheless, while the second order transition for the gas of 4NN exclusions is completely incompatible with even the modified version of the LL theory (in which the third order invariants are allowed), the case of $2 \mathrm{NN}$ is not nearly as bad. The LGW Hamiltonian for the lattice gas with 2NN exclusion $^{65}$ is

$$
\begin{aligned}
H= & \int \mathrm{d}^{2} \mathbf{x}\left\{\left(\nabla \psi_{1}\right)^{2}+\left(\nabla \psi_{2}\right)^{2}+r\left(\psi_{1}^{2}+\psi_{2}^{2}\right)+u\left(\psi_{1}^{2}+\psi_{2}^{2}\right)^{2}\right. \\
& \left.+v\left(\psi_{1}^{4}+\psi_{2}^{4}\right)\right\},
\end{aligned}
$$

where $\psi_{i}$ with $i=1,2$ are the two components of the order parameter belonging to the two dimensional irreducible representation of the space group $P 4 \mathrm{~mm}$.

In the absence of cubic anisotropy [last term of Eq. (11)) this Hamiltonian will not have a phase transition since the massless Goldstone excitations will destroy the long-range order at any finite temperature. Furthermore, Eq. (11) does not account for the topological defects (vortices) which are responsible for the phase transition in the real XY model ${ }^{85-87}$ and the appearance of a pseudo long-range order. Thus, in the passage from a microscopic Hamiltonian to the coarse grained LGW description, important physics has been left behind. $^{88,89}$ Clearly under such conditions no conclusions based on this LGW Hamiltonian can be trusted. Nevertheless, if we take the form of Eq. (11) more seriously than perhaps it deserves, the Ising criticality is not incompatible with its structure. Based on the general properties of $R G$ flows, we expect that Eq. (11) will have an Ising fixed point at which the isotropic fourth order invariant will vanish. At this fixed point, the LGW Hamiltonian will decouple into two Ising-type Hamiltonians for each component of the order parameter. The symmetry arguments, however, cannot tell us if the Ising fixed point is stable, or even if it is accessible to the renormalization group flow. Nevertheless, unlike the second order phase transition for $4 \mathrm{NN}$ exclusions, the Ising criticality for $2 \mathrm{NN}$ exclusions does not, in principle, invalidate the predictions of the LL theory. This not withstanding, it is quite surprising that in a multidimensional parameter space with an infinite number of fixed points (fixed lines) known to exist for the real $\mathrm{XY}$ model with cubic anisotropy, ${ }^{90}$ the $2 \mathrm{NN}$ model just happens to be in the region dominated by the Ising fixed point.

For lattice gas with 4NN exclusion the simulations find a continuous order-disorder transition in the Ising universality class. We note, however, that within a simulation it is impossible to be absolutely certain that the transition is not a very weakly first order as happens, for example, for the five state 
Potts model. There is, however, a big difference between $q$-state Potts models and hard core lattice gases studied in this paper. For Potts model, the mean-field theory ${ }^{84}$ predicts that all transitions for $q \geq 3$ are of first order. The fluctuations, however, modify the order of $q=3$ and 4 models to second, and turn $q=5$ model very weakly first order. ${ }^{83} \mathrm{Im}$ portance of fluctuations decreases for larger values of $q$ and the mean-field becomes progressively more accurate- the phase transitions for $q>4$ are all of first order. For lattice gases, on the other hand, mean-field theory (LL) predicts the transition to be of second order up to $2 \mathrm{NN}$ exclusions and first order afterwards. Indeed, we find continuous phase transitions for $1 \mathrm{NN}$ and $2 \mathrm{NN}$ exclusions and a first order transition for $3 \mathrm{NN}$. Unlike the case of Potts model, however, the agreement with the mean-field theory is broken precisely at $4 \mathrm{NN}$ and re-established again for $5 \mathrm{NN}$. There are no precursor fluctuation induced second order phase transitions (such as $q=3$ and 4 for Potts model) which would suggest that 4NN might have a weakly first order transition instead of a second order one. Furthermore, the critical exponents place $4 \mathrm{NN}$ directly into the Ising universality class. Thus, it would seem like too much of a coincidence for the Ising criticality to be only a crossover on the way to an underlying first order phase transition. We conjecture, in fact, that $4 \mathrm{NN}$ is not the only continuous transition for higher order exclusions. This conjecture is based on the observation that as the exclusion region grows, the system approaches a continuum limitlattice becomes irrelevant. For continuum 2D systems melting, however, is driven by the topological defects (dislocations) and is of second (actually infinite) order, belonging to the Kosterlitz-Thouless universality class. ${ }^{85-87,91-93}$ Clearly, this cannot be reconciled with the LL prediction of a first order transition for all exclusions above $2 \mathrm{NN}$. Thus, there must be other lattice gases with exclusions larger than $5 \mathrm{NN}$ which will also exhibit a continuous phase transition. The only way to fit this into LL theory is to relax the so called "Lifshitz rule" which forbids the representations for which the antisymmetric part of their direct product contains a vector representation. This, however, would completely destroy the LL theory since the number of possible representations which can then be used to construct an invariant LGW Hamiltonian will become infinite.

It is very difficult to understand the mechanism which changes a phase transition from first order, predicted by a mean-field theory, to second order when the fluctuations are taken into account. In two dimensions, however, this seems to be a very common occurrence as is demonstrated by the 3 and 4 state Potts models which undergo continuous transitions in spite of third order invariants present in their meanfield free energies. In this work we find that another mechanism is also possible - the fluctuations can invalidate the Lifshitz condition, transforming a first order phase transition into a fluctuation induced second order one. In view of our conjecture that $1 \mathrm{NN}, 2 \mathrm{NN}$, and $4 \mathrm{NN}$ are not the only lattice gases which undergo a continuous phase transition, it will be very curious to see what other higher order exclusions will also exhibit a continuous transition and what distinguishes these systems from the ones which undergo a discontinuous order-disorder phase transition.

\section{ACKNOWLEDGMENTS}

The authors would like to acknowledge J. A. Plascak for many interesting conversations and several helpful suggestions. This work was supported in part by the Brazilian science agencies CNPq, CAPES, and FAPERGS. H.C.M.F acknowledges the Universitat de Barcelona during his stay within the CAPES/MECD agreement.

${ }^{1}$ T. Hill, Statistical Mechanics (McGraw-Hill, New York, 1956).

${ }^{2}$ F. Ritort and P. Sollich, Adv. Phys. 52, 219 (2003).

${ }^{3}$ D. Gaunt and M. Fisher, J. Chem. Phys. 43, 2840 (1965).

${ }^{4}$ D. M. Burley, in Phase Transitions and Critical Phenomena, edited by C. Domb and M. S. Green (Academic, London, 1972), Vol. 2, Chap. 9, pp. 329-374.

${ }^{5}$ G. S. Rushbrooke and H. I. Scoins, Proc. R. Soc. London, Ser. A 230, 74 (1955).

${ }^{6}$ L. Lafuente and J. Cuesta, J. Chem. Phys. 119, 10832 (2003).

${ }^{7}$ D. Frenkel and B. Smit, Understanding Molecular Simulation (Academic, New York, 2000).

${ }^{8}$ A. Ferrenberg and R. Swendsen, Phys. Rev. Lett. 61, 2635 (1988).

${ }^{9}$ M. Newman and G. Barkema, Monte Carlo Methods in Statistical Physics (Oxford University Press, New York, 1999).

${ }^{10}$ W. Janke, in Computer Simulations of Surfaces and Interfaces, Proceedings of the NATO Advanced Study Institute Vol. 114, edited by B. Dünweg, D. P. Landau, and A. I. Milchev (Kluwer, Dordrecht, 2003), pp. $137-157$.

${ }^{11}$ A. Bellemans and R. Nigam, J. Chem. Phys. 46, 2922 (1967).

${ }^{12}$ M. E. Fisher, J. Math. Phys. 4, 278 (1963).

${ }^{13}$ R. J. Baxter, I. G. Enting, and S. K. Tsang, J. Stat. Phys. 22, 465 (1980).

${ }^{14}$ A. Baram and M. Fixman, J. Chem. Phys. 101, 3172 (1994).

${ }^{15}$ H. N. V. Temperley, Proc. Phys. Soc. London 80, 813 (1962).

${ }^{16}$ L. K. Runnels, Phys. Rev. Lett. 15, 581 (1965).

${ }^{17}$ L. K. Runnels and L. L. Combs, J. Chem. Phys. 45, 2482 (1966).

${ }^{18}$ F. H. Ree and D. A. Chesnut, J. Chem. Phys. 45, 3983 (1966).

${ }^{19}$ A. Bellemans and R. K. Nigam, Phys. Rev. Lett. 16, 1038 (1966).

${ }^{20}$ R. M. Nisbet and I. E. Farquhar, Physica (Amsterdam) 76, 259 (1974)

${ }^{21}$ D. W. Wood and M. Goldfinch, J. Phys. A 13, 2781 (1980).

${ }^{22}$ W. Kinzel and M. Schick, Phys. Rev. B 24, 324 (1981).

${ }^{23}$ P. A. Pearce and K. A. Seaton, J. Stat. Phys. 53, 1061 (1988).

${ }^{24}$ W. Guo and H. W. J. Blöte, Phys. Rev. E 66, 046140 (2002).

${ }^{25}$ Z. Ràcz, Phys. Rev. B 21, 4012 (1980).

${ }^{26}$ C.-K. Hu and C.-N. Chen, Phys. Rev. B 43, 6184 (1991).

${ }^{27}$ K. Binder and D. Landau, Phys. Rev. B 21, 1941 (1980).

${ }^{28}$ H. Meirovitch, J. Stat. Phys. 30, 681 (1983).

${ }^{29}$ C.-K. Hu and K.-S. Mak, Phys. Rev. B 39, 2948 (1989).

${ }^{30}$ T. M. Haas, J. Stat. Phys. 54, 201 (1989).

${ }^{31}$ A. Yamagata, Physica A 222, 119 (1995).

${ }^{32}$ J. R. Heringa and H. W. J. Blöte, Physica A 232, 369 (1996).

${ }^{33}$ J. R. Heringa and H. W. J. Blöte, Physica A 251, 224 (1998).

${ }^{34}$ D.-J. Liu and J. W. Evans, Phys. Rev. B 62, 2134 (2000).

${ }^{35}$ D. M. Burley, Proc. Phys. Soc. London 77, 451 (1961).

${ }^{36}$ L. K. Runnels, J. Math. Phys. 8, 2081 (1967).

${ }^{37}$ G. W. Woodbury, Jr., J. Chem. Phys. 47, 270 (1967).

${ }^{38}$ A. Robledo and C. Varea, J. Stat. Phys. 63, 1163 (1991).

${ }^{39}$ H. Hansen-Goos and M. Weigt, J. Stat. Mech.: Theory Exp. 2005, P04006 (2005).

${ }^{40}$ L. Lafuente and J. Cuesta, Phys. Rev. E 68, 066120 (2003).

${ }^{41}$ L. K. Runnels, in Phase Transitions and Critical Phenomena, edited by C. Domb and M. S. Green (Academic, London, 1972), Vol. 2, Chap. 8, pp. 305-328.

${ }^{42}$ R. J. Baxter, Ann. Comb. 3, 191 (1999).

${ }^{43}$ P. Fendley, K. Schoutens, and H. van Eerten, J. Phys. A 38, 315 (2005).

${ }^{44}$ G. E. Murch, Philos. Mag. A 44, 699 (1981).

${ }^{45}$ D. K. Chaturvedi, Phys. Rev. B 34, 8080 (1986).

${ }^{46}$ P. Meakin, J. L. Cardy, E. Loh, and D. J. Scalapino, J. Chem. Phys. 86, 2380 (1987)

${ }^{47}$ W. Ertel, K. Frobose, and J. Jackle, J. Chem. Phys. 88, 5027 (1988).

${ }^{48}$ J. Jäckie, K. Froböse, and D. Knödler, J. Stat. Phys. 63, 249 (1991).

${ }^{49}$ R. Dickman, J.-S. Wang, and I. Jensen, J. Chem. Phys. 94, 8252 (1991).

${ }^{50}$ J.-S. Wang, P. Nielaba, and V. Privman, Mod. Phys. Lett. B 7, 189 (1993).

${ }^{51}$ E. Eisenberg and A. Baram, Europhys. Lett. 44, 168 (1998). 
${ }^{52}$ R. Dickman, Phys. Rev. E 64, 016124 (2001).

${ }^{53}$ H. Hansen-Goos and M. Weigt, J. Stat. Mech.: Theory Exp. 2005, P08001 (2005).

${ }^{54}$ F. Q. Potiguar and R. Dickman, Eur. Phys. J. B 52, 83 (2006).

${ }^{55}$ D. S. Gaunt, J. Chem. Phys. 46, 3237 (1967).

${ }^{56}$ E. Cowley, J. Chem. Phys. 71, 458 (1979).

${ }^{57}$ C.-K. Hu and K.-S. Mak, Phys. Rev. B 42, 965 (1990).

${ }^{58}$ J. R. Heringa, H. W. J. Blöte, and E. Luijten, J. Phys. A 33, 2929 (2000).

${ }^{59}$ A. Z. Panagiotopoulos, J. Chem. Phys. 123, 104504 (2005).

${ }^{60}$ D. Landau and K. Binder, A Guide to Monte Carlo Simulations in Statistical Physics (Cambridge University Press, Cambridge, 2000).

${ }^{61}$ B. G. Baker, J. Chem. Phys. 45, 2694 (1966).

${ }^{62}$ F. Ree and D. Chesnut, Phys. Rev. Lett. 18, 5 (1967).

${ }^{63}$ M. L. Mehta, J. Chem. Phys. 60, 2207 (1974).

${ }^{64}$ R. Nisbet and I. Farquhar, Physica (Amsterdam) 73, 351 (1974).

${ }^{65}$ E. Domany, M. Schick, J. Walker, and R. Griffiths, Phys. Rev. B 18, 2209 (1978)

${ }^{66}$ E. Aksenenko and Y. Shulepov, J. Phys. A 15, 2515 (1982).

${ }^{67}$ P. A. Slotte, J. Phys. A 16, 2935 (1983).

${ }^{68}$ D. A. Huckaby, J. Stat. Phys. 33, 23 (1983).

${ }^{69}$ E. Aksenenko and Y. Shulepov, J. Phys. A 17, 2109 (1984).

${ }^{70}$ J. Amar, K. Kaski, and J. D. Gunton, Phys. Rev. B 29, 1462 (1984).

${ }^{71}$ J.-S. Wang, P. Nielaba, and V. Privman, Physica A 199, 527 (1993).

${ }^{72}$ C. Uebing, Physica A 210, 205 (1994).
${ }^{73}$ M. Schmidt, L. Lafuente, and J. Cuesta, J. Phys.: Condens. Matter 15, 4695 (2003)

${ }^{74}$ N. Bartelt and T. Einstein, Phys. Rev. B 30, 5339 (1984).

${ }^{75}$ E. Müller-Hartmann and J. Zittarz, Z. Phys. B 27, 261 (1977).

${ }^{76}$ O. J. Heilmann and E. Praestgaard, J. Phys. A 7, 1913 (1974).

${ }^{77}$ J. Orban and D. van Belle, J. Phys. A 15, L501 (1982).

${ }^{78}$ A. Bellemans and J. Orban, Phys. Rev. Lett. 17, 908 (1966).

${ }^{79}$ R. M. Nisbet and I. E. Farquhar, Physica (Amsterdam) 76, 283 (1974).

${ }^{80}$ E. Eisenberg and A. Baram, Europhys. Lett. 71, 900 (2005).

${ }^{81}$ C. K. Hall and G. Stell, Phys. Rev. A 7, 1679 (1973).

${ }^{82}$ M. E. Fisher and A. Nihat Berker, Phys. Rev. B 26, 2507 (1982).

${ }^{83}$ R. J. Baxter, J. Phys. C 6, L445 (1973).

${ }^{84}$ L. Mittag and M. J. Stephen, J. Phys. A 7, L109 (1974).

${ }^{85}$ J. M. Kosterlitz and D. J. Thouless, J. Phys. C 6, 1181 (1973).

${ }^{86}$ V. L. Berezinskii, Zh. Eksp. Teor. Fiz. 61, 1144 (1971).

${ }^{87}$ V. L. Berezinskii, Sov. Phys. JETP 34, 610 (1971).

${ }^{88}$ Y. Levin and K. A. Dawson, Phys. Rev. A 42, 3507 (1990).

${ }^{89}$ Y. Levin, Phys. Rev. B 43, 10876 (1991).

${ }^{90}$ J. V. José, L. P. Kadanoff, S. Kirkpatrick, and D. R. Nelson, Phys. Rev. B 16, 1217 (1977).

${ }^{91}$ D. R. Nelson, Phys. Rev. B 18, 2318 (1978).

${ }^{92}$ D. R. Nelson and B. I. Halperin, Phys. Rev. B 19, 2457 (1979).

${ }^{93}$ A. P. Young, Phys. Rev. B 19, 1855 (1979). 